\title{
Applications of Nanoparticles for Reactive Oxygen Species (ROS) Scavenging in Photosynthetic Systems
}

\author{
A. A. Boghossian ${ }^{\mathrm{a}}$ \\ a Institute of Chemical Sciences and Engineering, École Polytechnique Fédérale de \\ Lausanne (EPFL), 1015 Lausanne, Switzerland
}

Under aerobic conditions, excessive illumination results in the photogeneration of reactive oxygen species (ROS) responsible for protein damage and photo-inhibition during photosynthesis. We explore the effects of nanoceria $(\mathrm{NC})$, a regenerative ROS scavenger, on chloroplasts isolated from plant leaves. The addition of dextran-wrapped nanoceria ( $\mathrm{dNC}$ ) was shown to decrease ROS concentrations in photoactive chloroplast solutions. We also explore the application of single-walled carbon nanotubes (SWCNTs) as vehicles for nanoparticle uptake inside not only chloroplasts, but also whole plant leaves of $S$. oleracea.

\section{Introduction}

Dynamic cell processes occur at the nanoscale, and nanomaterials offer a quintessential opportunity to not only understand, but also engineer, cellular processes. By definition, nanomaterials contain at least one dimension below $100 \mathrm{~nm}$. Provided the microscale dimensions of cells, nanomaterials can readily interface with the building blocks of life by either adhering to cell surfaces or entering cells. Recent endeavors have focused on engineering nanomaterials that demonstrate desirable properties for drug delivery and biosensing applications. These nanoparticles have been used to not only provide spatiotemporal sensing inside and outside of cells, but also behave as vehicles for transporting both beneficial and detrimental drugs to targeted cells and targeted regions within the cell (1-3).

Such studies have been expanded to explore nanoparticle interactions with not only mammalian cells, but also plant cells. Rising concerns over the environmental impact of nanoparticles have fueled the surge in nano-botanic research (4). Although most nanobotanic studies focus on environmental effects such as plant cytotoxicity and germination, recent studies are focusing on developing nanomaterials specifically engineered to supplement and, in some cases, improve natural plant function. For instance, light absorption by plants is controlled by light-harvesting proteins and pigments inside the plant cell that are largely limited to the visible region of the spectrum. These pigments have limited absorption between $500-600 \mathrm{~nm}$, a range that overlaps with the region of the solar spectrum with maximum solar irradiance. Nanoparticles such as single-walled carbon nanotubes (SWCNTs), on the other hand, not only absorb light throughout the solar spectrum, but also demonstrate tunable optoelectronic properties desirable for solar energy conversion applications (5). These complementary advantages make nanoparticles promising candidates for augmenting photosynthetic systems. 
In addition to desirable optoelectronic properties, nanoparticles offer another inherent advantage for enhancing natural plant function: high surface area to volume ratios. The surface area to volume ratio of materials scales inversely with radius, with surface effects dominating at the nanoscale. The relatively high surface areas of nanoparticles are particularly beneficial to surface-controlled catalytic reactions. Among the many surfacemediated, biological reactions of interest is reactive oxygen species (ROS) scavenging. ROS serves as a signaling molecule in both mammalian and plant cells. ROS sensors have been used to image the spatiotemporal distribution of signaling molecules of living cells while ROS scavengers can modulate the state of metabolically stressed systems.

This article focuses on the application of a nanoparticle-based ROS scavenging platform to photosynthetic systems. We specifically explore the use of nanoceria (NC) as a regenerative, ROS scavenger inside chloroplasts, plant organelles where photosynthesis takes place (6).

\section{Background}

Pigments such as chlorophyll are responsible for absorbing the light that drives photosynthesis. These pigments have been engineered by nature not necessarily to maximize the amount of light absorbed by plant, but to ensure the survivability of the plant. In fact, up to $80 \%$ of the sunlight is either reflected or actively dissipated as heat or fluorescence in order to protect the plant from photo-induced protein damage and subsequent cellular inactivation. The absorption of excess photons results in the excitation of chlorophyll from the ground state to the singlet state. This short-lived singlet state is quickly converted to the triplet state, which readily reacts with molecular oxygen to produce singlet oxygen. Singlet oxygen subsequently reacts with neighboring proteins such as the D1 protein, which is essential for photosynthesis, to induce photodamage. Other ROS, such as superoxide, that also contribute to photodamage are formed through the over-reduction of the photosynthetic proteins, PSII and PSI.

To minimize the detrimental oxidative effects of ROS generation, plants have evolved a natural mechanism for ROS scavenging called the water-water cycle. The water-water cycle converts photodamaging ROS to water using the enzymes superoxide dismutase and ascorbate peroxidase. During the water-water cycle, ascorbate peroxidase undergoes oxidative damage in the presence of ROS. The damaged enzyme is then continually regenerated during the water-water cycle through a series of cyclical reactions. Prolonged periods of excessive illumination results in over-oxidative metabolic stresses beyond the regenerative capabilities of the water-water cycle that contribute to irreversible photodamage.

One approach to augmenting the water-water cycle to minimize oxidative plant stress is to couple photosynthetic machinery with synthetic, regenerative ROS scavengers such as nanoceria (NC) that are less susceptible to photodamage (7). $\mathrm{NC}$ are $\mathrm{CeO}_{2}$ nanoparticles that consist of $\mathrm{Ce}^{4+}$ in the bulk phase. When particle sizes are below $5 \mathrm{~nm}$, the lattice strain introduces the formation of defect sites that contain $\mathrm{Ce}^{3+}$. These defect sites readily react with oxidizing agents such as ROS, effectively scavenging ROS to form $\mathrm{Ce}^{4+}$. Though the conversion of $\mathrm{Ce}^{3+}$ to $\mathrm{Ce}^{4+}$ eliminates the defect site, the resulting increase in the lattice stresses of the nanoparticle regenerates the surface defect elsewhere 
on the particle. As such, NC behaves as a regenerative ROS scavenger that can supplement the water-water cycle.

The ability of NC to regeneratively scavenge for ROS is dependent on maintaining a balance between the $\mathrm{Ce}^{3+}$ and $\mathrm{Ce}^{4+}$ oxidation states. Under strongly basic conditions, $\mathrm{Ce}^{3+}$ and $\mathrm{Ce}^{4+}$ are converted to $\mathrm{Ce}(\mathrm{OH})_{3}$ and $\mathrm{Ce}(\mathrm{OH})_{4}$, respectively, which precipitate out of solution. Under strongly acidic conditions, $\mathrm{Ce}^{3+}$ is irreversibly converted to $\mathrm{Ce}^{4+}$ and, because a catalytic amount of $\mathrm{Ce}^{3+}$ is necessary for ROS scavenging, the catalyst becomes deactivated. Therefore, nanoparticle activity is strongly $\mathrm{pH}$ dependent, and the $\mathrm{pH}$ range at which the nanoparticles are able to scavenge for ROS depends on the polymer wrapping of the NC. For biological applications, dextran-wrapped NC (dNC) provide effective scavenging at physiological $\mathrm{pHs}(\mathrm{pH} \sim 7)$.

\section{Experimental}

The dNC was synthesized according to previous studies (7). Briefly, an aqueous solution of dextran and cerium nitrate was rigorously stirred for 2 hours, and ammonium hydroxide was added to achieve a final $\mathrm{pH}$ of 7.3-7.6. The size distribution of the nanoparticles was verified using atomic force miscroscopy (AFM) and transmission electron microscopy (TEM). Absorption and x-ray photoelectron spectroscopy (XPS) measurements verify the presence of both the $\mathrm{Ce}^{3+}$ and $\mathrm{Ce}^{4+}$ oxidation states.

Two dyes were used to quantify ROS scavenging in chloroplasts: 2',7'dichlorodihydrofluorescein diacetate $\left(\mathrm{H}_{2} \mathrm{DCF}-\mathrm{DA}\right)$ and 2,3-bis(2-methoxy-4-nitro-5sulfophenyl)-2H-tetrazolium-5-carboxanilide sodium salt (XTT). The $\mathrm{H}_{2}$ DCF-DA is commonly used to nonspecifically monitor ROS generation in a variety of biological systems. Oxidative cleavage of the acetate groups by ROS causes the dye to fluoresce at $520 \mathrm{~nm}$ upon $485 \mathrm{~nm}$ excitation. Superoxide concentrations were also specifically monitored using XTT, which absorbs at $470 \mathrm{~nm}$ when reduced by superoxide.

In addition to studying dNC efficacy, dNC uptake and localization was also explored using confocal fluorescence microscopy. For these studies, the $\mathrm{dNC}$ was prepared as described above, except using Alexa Fluor 488-labelled dextran.

\section{Results and Discussion}

The dyes $\mathrm{H}_{2}$ DCF-DA and XTT were used to monitor the effects of dNC on ROS scavenging in chloroplast solutions, and the results of these measurements are summarized in Figure 1. As shown in the figure, $\mathrm{H}_{2} \mathrm{DCF}-\mathrm{DA}$ fluorescence and XTT absorption decrease with the addition of $\mathrm{dNC}$, suggesting an overall decrease in ROS concentrations. Control measurements in the dark verify that ROS generation is photoinduced. 


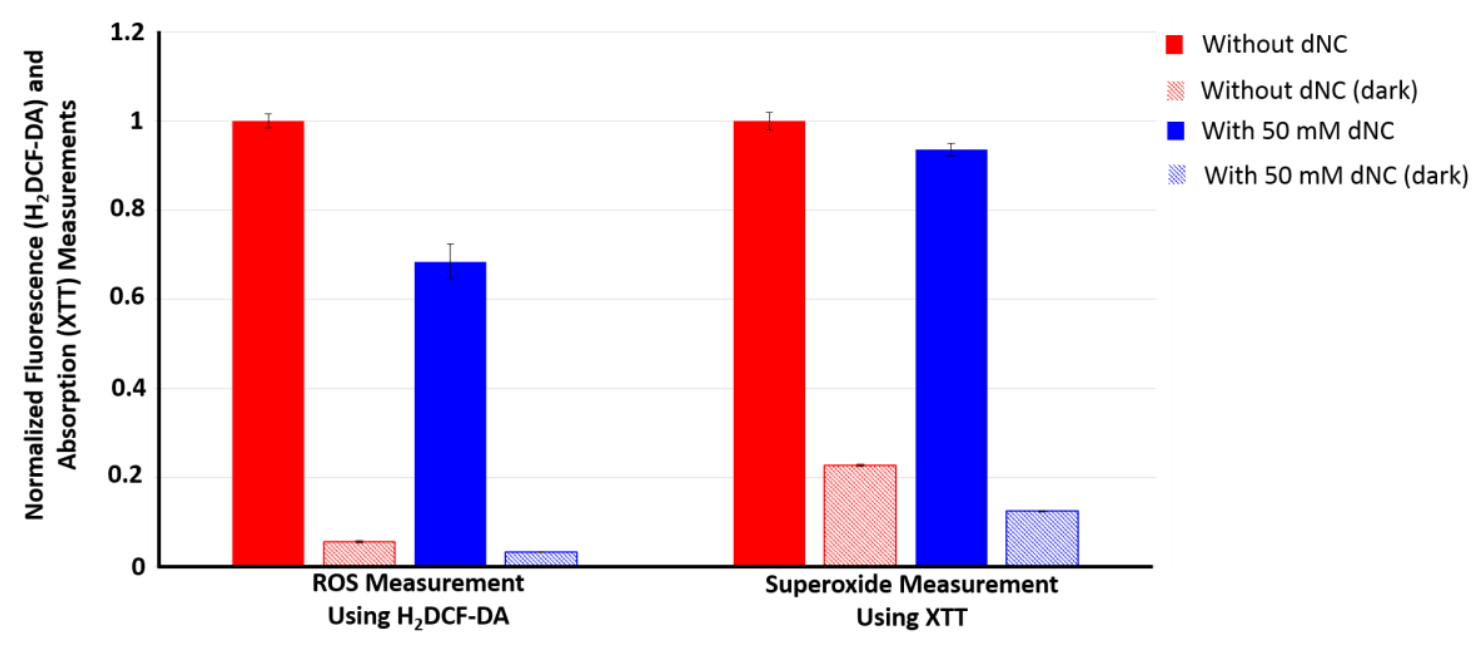

Figure 1. ROS and superoxide concentrations of chloroplast solutions were measured using $\mathrm{H}_{2}$ DCF-DA fluorescence (ex. $485 \mathrm{~nm}$, em. $520 \mathrm{~nm}$ ) and XTT (abs. $470 \mathrm{~nm}$ ). Measurements were taken for both illuminated chloroplast solutions (solid) and chloroplast solutions incubated in the dark (patterned). The $\mathrm{H}_{2}$ DCF-DA measurements were taken after a 6-hour incubation period, and XTT measurements were taken after a 2hour incubation period. The fluorescence and absorption values were normalized to the corresponding measurements of chloroplast solutions in the absence of dNC. (Figure modified from ref. 6).

To evaluate dNC localization in the chloroplast, confocal fluorescence microscopy was used to image chloroplasts with fluorescently-labeled dNC. The confocal images shown in Figure 2 show no overlap in fluorescence between the labelled dNC and the chloroplasts, indicating that the chloroplasts do not uptake the nanoparticles. Previous studies have shown that cellular uptake is strongly dependent on the zeta potential of the nanoparticle's polymer wrapping, with nanoparticle uptake facilitated by wrappings that have either relatively positive or negative zeta potentials. Recent measurements have explored the effects of different $\mathrm{NC}$ polymer wrappings on chloroplast uptake, verifying NC uptake with positively and negatively charged polymer potentials (8).

In addition to NC, recent studies have also explored SWCNT uptake by chloroplasts. The polymer wrappings were also shown to modulate the intake of SWCNTs and SWCNT-based complexes. Conjugating poly(acrylic acid) (PAA)-wrapped NC to SWCNTs allowed the chloroplast to take up the nanoparticles, enabling effective ROS scavenging inside the organelle (8). 

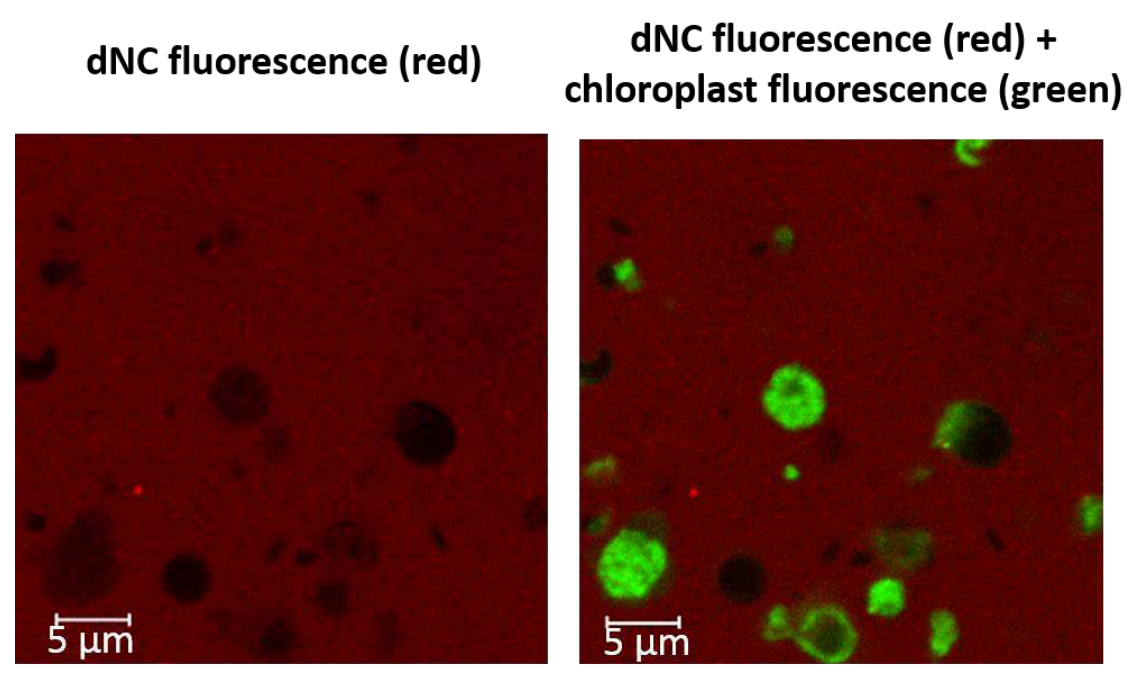

Figure 2. Confocal fluorescence images were taken of Alexa Fluor 488-labeled dNC (ex. $488 \mathrm{~nm}$, em. 500-530 nm) and chloroplasts (ex. $488 \mathrm{~nm}$, em. 660-700 nm). (Figure modified from ref. 6).

These platforms have been applied to not only chloroplasts, but also whole plant leaves. For example, phenoxy dextran-wrapped SWCNTs were taken up by cut $S$. oleracea leaves via transpiration. SWCNT uptake was verified through raman spectroscopy (Figure 3). These preliminary raman measurements indicate that the SWCNTs are mostly concentrated at the leaf petiole. Such platforms exemplify the application of SWCNTs as possible drug delivery vehicles and biosensors for whole plant leaves.

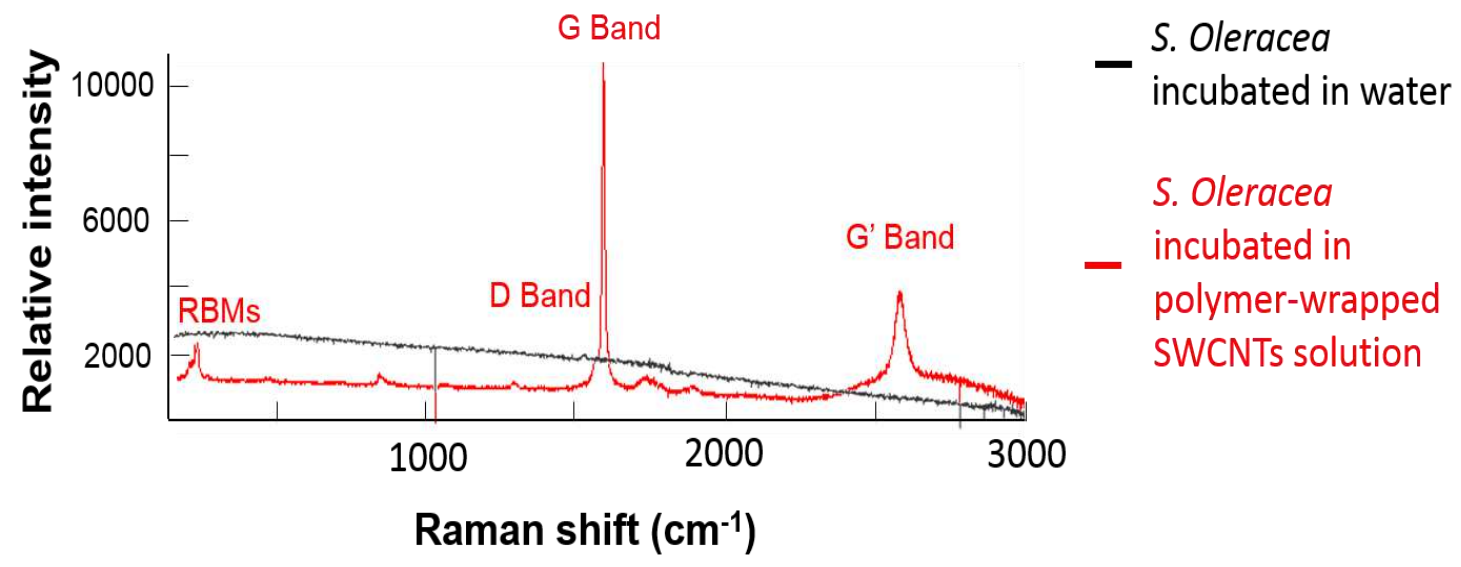

Figure 3. Raman measurement of the petiole of a S. oleracea leaf after transpiration in the absence and presence of phenoxy dextran-wrapped SWCNTs. Leaves exposed to the polymer-wrapped SWCNTs exhibit the expected spectral characteristics of SWCNTs. 


\section{Acknowledgments}

The work performed at the Massachusetts Institute of Technology (MIT) was supported by a grant from the U.S. Department of Energy (grant no. ER46488), as well as fellowship support from the National Defense Science and Engineering Graduate (NDSEG) Fellowship, the TUBITAK 2211 and 2214 research fellowship program, the METU-DPT-OYP program, the National Science Foundation (NSF) Graduate Fellowship and the National Science Foundation Postdoctoral Research Fellowship in Biology under Grant No. 1103600.

\section{References}

1. A. Z. Wang, R. Langer, and O. C. Farokhzad in Annual Review of Medicine Vol. 63, C. T. Caskey, C. P. Austin, and J. A. Hoxie, Editors, p. 185, Annual Reviews, Palo Alto (2012).

2. S. Diao, G. S. Hong, J. T. Robinson, L. Y. Jiao, A. L. Antaris, J. Z. Wu, C. L. Choi, and H. J. Dai, JACS, 134(41), 16971 (2012).

3. R. M. Williams, J. Shah, B. D. Ng, D.R. Minton, L.J. Gudas, C. Y. Park, and D. A. Heller, Nano Lett., 15(4), 2358 (2015).

4. E. Navarro, A. Baun, R. Behra, N. B. Hartmann, J. Filser, A. J. Miao, A. Quigg, P. H. Santschi, and L. Sigg, Ecotoxicology, 17(5), 372 (2008).

5. M. S. Arnold, J. L. Blackburn, J. J. Crochet, S. K. Doorn, J. G. Duque, A. Mohite, and H. Telg, Phys. Chem. Chem. Phys., 15(36), 14896 (2013).

6. A. A. Boghossian, F. Sen, B. M. Gibbons, S. Sen, S. M. Faltermeier, J. P. Giraldo, C. T. Zhang, J. Zhang, D. A. Heller, and M. S. Strano, Adv. Energy Mater., 3(7), 881 (2013).

7. J. Chen, S. Patil, S. Seal, and J. F. McGinnis, Nat. Nanotech., 1, 142 (2006).

8. J. P. Giraldo, M. P. Landry, S. M. Faltermeier, T. P. McNicholas, N. M. Iverson, A. A. Boghossian, N. F. Reuel, A. J. Hilmer, F. Sen, J. A. Brew, and M. S. Strano, Nature Mater., 13, 400 (2014). 